\title{
Evaluation of Reporting Quality in Randomised Controlled Trials of Acupuncture for Acute Herpes Zoster by the CONSORT Statement and STRICTA Guidelines
}

\author{
Guifeng Qian $\mathbb{D}^{\mathbb{D}},{ }^{1,2}$ Jingchun Zeng, ${ }^{3}$ Liming Lu $\mathbb{D}^{4},{ }^{4}$ Wenya Pei $\mathbb{D}^{5},{ }^{5}$ Kun Liu, ${ }^{6}$ Zhenke Luo $\mathbb{D}^{6}$, \\ Yalin She, ${ }^{6}$ Pande Zhang $\left(\mathbb{D},{ }^{2}\right.$ and Guohua Lin $\mathbb{1}^{3}$ \\ ${ }^{1}$ Department of Neurology, Zhujiang Hospital, Southern Medical University, Guangzhou 510282, China \\ ${ }^{2}$ Department of Rehabilitation, The First People's Hospital of Foshan (Affiliated Foshan Hospital of Sun Yat-sen University), \\ Foshan 528000, China \\ ${ }^{3}$ Department of Acupuncture, The First Affiliated Hospital, Guangzhou University of Chinese Medicine, \\ Guangzhou 510405, China \\ ${ }^{4}$ Clinical Research and Data Center, Medical College of Acu-Moxi and Rehabilitation, \\ Guangzhou University of Chinese Medicine, Guangzhou 510405, China \\ ${ }^{5}$ The First Affiliated Hospital, Sun Yat-Sen University, Guangzhou 510080, China \\ ${ }^{6}$ The First Clinical Medical College, Guangzhou University of Chinese Medicine, Guangzhou 510405, China
}

Correspondence should be addressed to Pande Zhang; 2930502616@qq.com and Guohua Lin; tcmlin-801@163.com

Received 20 August 2019; Accepted 16 January 2020; Published 18 February 2020

Academic Editor: Maruti Ram Gudavalli

Copyright ( 2020 Guifeng Qian et al. This is an open access article distributed under the Creative Commons Attribution License, which permits unrestricted use, distribution, and reproduction in any medium, provided the original work is properly cited.

\begin{abstract}
Objective. To explore the methods for improving the reporting quality of randomised controlled trials (RCTs) on acupuncture through evaluating the reporting quality in RCTs of acupuncture for acute herpes zoster by the CONSORT statement and STRICTA guidelines. Methods. English and Chinese databases were searched from database creation until October 2018 and updated to July 2019. The basic characteristics and methodological quality of the literatures included were evaluated based on the CONSORT statement and the STRICTA guidelines. Descriptive statistical analysis was used in this study. The agreement between the two researchers of all items was calculated by Cohen's kappa statistics. Results. A total of 40 RCTs were included. Based on the CONSORT statement, items "Background," "Randomised" in the title or abstract," "Statistical methods," and "Outcomes and estimation" were good reporting, with positive rates $>80 \%$. However, the quality of reporting in items "Sample size," "Allocation concealment," "Implementation," "Blinding," "Flow chart," "Intent-to-treat analysis," "Ancillary analyses," and "Clinical Trials Register" was very poor, with positive rates $<10 \%$. Based on the STRICTA guidelines, good reporting existed in items "Acupuncture rational," "Points used," "Needle stimulation," "Needle retention time," "Course of treatment," "Control intervention," and "Treatment frequency," with positive ratings $>80 \%$. The reporting quality of items "Numbers of needles inserted," "Depth of insertion," "Responses elicited," and "Practitioner background" was lower, with positive rates $<50 \%$. The agreement of most items was judged as moderate, substantial, or good.Conclusion. The reporting quality of RCTs in acupuncture for acute HZ is generally inadequate. It is necessary that researchers and journal editors learn and raise the adoption of the CONSORT statement and STRICTA guidelines to enhance the reporting quality of the RCTs in acupuncture.
\end{abstract}

\section{Introduction}

Herpes zoster (HZ) is caused by activation of varicella-zoster virus resulting in sensory ganglia infection [1]. The major symptoms of acute $\mathrm{HZ}$ are the severe pain, characteristic rash, and distinctive distribution, which reduce quality of life [2]. HZ can occur in all age groups especially in elderly. The incidence rate of HZ rises sharply from the age of 60 and reaches 11 cases per 1000 at the age of 90 , roughly half of those who reach 85 years old will develop HZ [3]. The 
standard treatment for HZ is antiviral therapy, despite the progress of the vaccine advanced. Research has shown that the effect of $\mathrm{HZ}$ vaccine lasted only 8 years [4], so adults vaccinated before the age of 60 years might not be protected at the highest risk of HZ. The efficacy of the vaccine still needs further research and improvement [5]. More and more physicians and patients have accepted acupuncture as a complement therapy for a series of diseases especially pain in western countries. A cross-sectional study investigated that the pain was the top acupuncture indications and was nearly half of the clinical problem acupuncturists encountered in the US clinics [6]. Acupuncture was reported for the treatment of HZ [7-10], which showed that it relieves pain of $\mathrm{HZ}$, shortens the healing of the rash, and reduces the occurrence of postherpetic neuralgia (PHN). But no studies have evaluated the reporting quality of these papers.

The "Consolidated Standards of Reporting Trials" (CONSORT) guidelines, updated in 2001 and 2010, provided checklists for the author to write papers for publication $[11,12]$. It is widely used to evaluate the reporting quality of clinical trials today. The "Standards for Reporting Interventions in Controlled Trials of Acupuncture" (STRICTA) guidelines, extending the CONSORT guidelines, is to improve the reporting quality of clinical trials for acupuncture $[13,14]$. A series of studies showed they help readers obtain information about research design, intervention implementation, data analysis, and so on [15-18]. They have positively impacted on reporting quality of the trials. Based on the above guidelines, this study was conducted to clarify the reporting quality of acupuncture for acute $\mathrm{HZ}$ by CONSORT statement and STRICTA guidelines.

\section{Methods}

2.1. Search Strategy. The databases Ovid Embase, Ovid Medline, Cochrane Library, WanFang database, Chongqing Weipu (VIP), and China National Knowledge Infrastructure (CNKI) were searched from creation until October 2018 and updated it to July 2019. The following search terms were used in Chinese and English: (Acupuncture OR Acupuncture therapy OR Electro-acupuncture OR Auricular acupuncture OR Scalp acupuncture OR Fire needle OR Warming needle OR Needle OR Acupuncture point OR Meridian OR Acupoint OR Jingluo) AND (Herpes zoster OR Shingles OR Varicella-zoster virus). All articles were published either in Chinese or English. The details of the search strategy can be found in Supplementary File 1.

\subsection{Inclusion and Exclusion Criteria}

2.2.1. Types of Studies. We included any clinical reports assessing the efficacy of acupuncture in the treatment of acute HZ and mentioning "Randomised Controlled Trials" in the title, abstract, or text, regardless of blinding, implementation, or allocation concealment were used or not in the study. Nonrandomized papers, review, abstract, and case report were included. Any studies with no available data or outcomes for extraction have been excluded.
2.2.2. Types of Participants. Patients of any gender, age, or ethnicity with $\mathrm{HZ}$ in the acute phase were eligible. Herpes zoster is diagnosed clinically by the pain, characteristic rash, and distinctive distribution [19]. The acute phase of $\mathrm{HZ}$ usually occurs during the 30 days after the onset of the characteristic rash [20]. Patients of any types of $\mathrm{HZ}$ with the exception of visceral $\mathrm{HZ}$, meningeal $\mathrm{HZ}$, and $\mathrm{PHN}$ were qualified.

2.2.3. Types of Interventions. Different forms of acupuncture techniques or needle were included consisting of manual acupuncture, electroacupuncture, fire or warm needle, scalp acupuncture, and auricular acupuncture, except laser acupuncture and acupressure. The intervention of the experimental group was acupuncture alone or acupuncture combined with medication same as the control group. The control group used placebo, no treatment, antivirals, neurotrophic drugs, corticosteroids, and analgesics, while acupuncture, traditional Chinese medicine, proprietary Chinese medicine, moxibustion, cupping, or physical therapy did not contained.

2.3. Selection of Reports. Firstly, two researchers (GQ and JZ) reviewed the title and abstract of citations retrieved and read the full report through NoteExpress separately. Any article that does not meet incorporate criteria or match exclusions standards moved into the specified folder with different labels in NoteExpress. For example, nonrandomized controlled trials, case report, animal experiments, comments, and reviews are removed in the specified folder. The entire team discusses any uncertain literature and makes the final decision.

2.4. Data Extraction. Two researchers (WP and KL) used the EpiData software to selected information from the final documents after receiving training of research and statistical methods about the CONSORT and STRICTA checklists. The general information includes the title of the paper, the author, the year of publication, the name of the journal, the type of journal, and the language of the publication. Any information unmentioned was encoded as "not reported." Two researchers analyzed the full text of 40 articles and performed data extraction.

2.5. Assessment of Reporting Quality. We assessed the reporting quality of RCTs in acupuncture for acute $\mathrm{HZ}$ according to the CONSORT statement and STRICTA guidelines. Items of them can be found in Tables 1 and 2 . Every article was reviewed by two investigators (GQ and JZ). If report 0 indicates that the item is not explicitly stated, it is marked as 1 . The agreement between the two researchers been evaluated through calculating Cohen's kappa. Agreement was judged as good if $\kappa$ was $>0.8$, substantial if $0.6<\kappa \leq 0.8$, moderate if $0.4<\kappa \leq 0.6$, fair if $0.2<\kappa \leq 0.4$, and poor if $\kappa$ was $\leq 0.2$. Any disagreements or difficulties were solved through conversation in the process. 95\% CI and Cohen's kappa of each variable were performed by the 
TABLE 1: Reporting quality using items from the CONSORT statement ( $n=40$ studies).

\begin{tabular}{|c|c|c|c|c|c|c|}
\hline \multicolumn{2}{|c|}{$\begin{array}{c}\text { Reporting quality using items from the CONSORT } \\
\text { statement ( } n=40 \text { studies) }\end{array}$} & \multirow{2}{*}{$\begin{array}{c}\begin{array}{c}\text { No. of } \\
\text { positive trials }\end{array} \\
35\end{array}$} & \multirow{2}{*}{$\begin{array}{l}\% \\
88\end{array}$} & \multirow{2}{*}{$\begin{array}{l}95 \% \mathrm{CI} \\
77 \text { to } 99\end{array}$} & \multirow{2}{*}{$\begin{array}{c}\text { Cohen's } \\
\text { Kappa } \\
0.77\end{array}$} & \multirow{2}{*}{$\begin{array}{c}95 \% \mathrm{CI} \\
0.63 \text { to } 0.91\end{array}$} \\
\hline $\begin{array}{l}\text { "Randomised" in the title } \\
\text { or abstract }\end{array}$ & $\begin{array}{l}\text { "Randomised" in the title } \\
\text { or abstract }\end{array}$ & & & & & \\
\hline Background & $\begin{array}{l}\text { Adequate description of the } \\
\text { scientific background and explanation } \\
\text { of rationale }\end{array}$ & 36 & 90 & 80 to 99 & 0.84 & 0.72 to 0.96 \\
\hline Trial design & $\begin{array}{l}\text { Description of trial design (such } \\
\text { as parallel and factorial) including } \\
\text { the allocation ratio }\end{array}$ & 7 & 18 & 5 to 31 & 0.81 & 0.68 to 0.94 \\
\hline Participants & $\begin{array}{l}\text { Description of the eligibility criteria } \\
\text { for participants }\end{array}$ & 21 & 53 & 37 to 69 & 0.70 & 0.55 to 0.85 \\
\hline Interventions & $\begin{array}{l}\text { Details of the interventions intended } \\
\text { for each group(refer Table } 2)\end{array}$ & - & - & - & - & - \\
\hline Outcomes & $\begin{array}{l}\text { Definition of primary (and secondary } \\
\text { when appropriate) outcome measures }\end{array}$ & 31 & 78 & 64 to 92 & 0.59 & 0.43 to 0.75 \\
\hline Sample size & Description of sample size calculation & 2 & 5 & 0 to 12 & 1 & - \\
\hline & $\begin{array}{l}\text { (a) Method used to generate the random } \\
\text { allocation sequence }\end{array}$ & 13 & 33 & 18 to 48 & 0.70 & 0.55 to 0.85 \\
\hline Randomisation & $\begin{array}{l}\text { (b) Type of randomisation details of } \\
\text { any restriction }\end{array}$ & 0 & 0 & - & - & - \\
\hline Allocation concealment & $\begin{array}{l}\text { Description of the method used to } \\
\text { implement the random allocation } \\
\text { sequence assuring the concealment } \\
\text { until interventions were assigned }\end{array}$ & 0 & 0 & - & - & - \\
\hline Implementation & $\begin{array}{c}\text { Who generated the random allocation } \\
\text { sequence, who enrolled participants, } \\
\text { and who assigned participants } \\
\text { to interventions }\end{array}$ & 3 & 8 & 0 to 17 & 0.36 & 0.20 to 0.51 \\
\hline & $\begin{array}{l}\text { If done, who was blinded after } \\
\text { assignment to interventions }\end{array}$ & & & & & \\
\hline \multirow[t]{3}{*}{ Blinding } & (a) Participants & 0 & & & & \\
\hline & (b) Care providers & 0 & & & & \\
\hline & (c) Outcome assessors & 1 & 3 & 0 to 9 & 1 & - \\
\hline Statistical methods & $\begin{array}{c}\text { Description of the statistical methods } \\
\text { used to compare groups for primary } \\
\text { outcomes, subgroup analyses, or } \\
\text { adjusted analyses }\end{array}$ & 34 & 85 & 73 to 97 & 0.47 & 0.31 to 0.63 \\
\hline Flow chart & $\begin{array}{l}\text { Details on the flow of participants } \\
\text { through each stage of the trials }\end{array}$ & 2 & 5 & 0 to 12 & 1 & - \\
\hline Recruitment & $\begin{array}{l}\text { Dates defining the periods of } \\
\text { recruitment and follow-up }\end{array}$ & 27 & 68 & 53 to 83 & 0.58 & 0.41 to 0.74 \\
\hline Baseline data & $\begin{array}{l}\text { An outline of baseline demographic } \\
\text { and clinical characteristics of each group }\end{array}$ & 29 & 73 & 58 to 88 & 0.78 & 0.64 to 0.92 \\
\hline Intent-to-treat analysis & $\begin{array}{l}\text { Number of participants in each } \\
\text { group included in each analysis and } \\
\text { whether it was done by } \\
\text { "intention-to-treat" }\end{array}$ & 0 & 0 & - & 1 & - \\
\hline Outcomes and estimation & $\begin{array}{l}\text { For each primary and secondary } \\
\text { outcome, summary of results for } \\
\text { each group was given as well as } \\
\text { the estimated effect size and } \\
\text { its precision (e.g., } 95 \% \mathrm{CI} \text { ) }\end{array}$ & 40 & 100 & - & 1 & - \\
\hline Ancillary analyses & $\begin{array}{l}\text { Clear statement of whether } \\
\text { subgroup/adjusted analyses } \\
\text { were prespecified or exploratory }\end{array}$ & 0 & 0 & - & 1 & - \\
\hline Adverse events & $\begin{array}{l}\text { Description of all important } \\
\text { adverse events in each group }\end{array}$ & 7 & 18 & 5 to 30 & 0.92 & 0.83 to 1 \\
\hline Clinical trials register & Whether to conduct clinical trial registration & 1 & 3 & 0 to 9 & 1 & - \\
\hline Funding & Fund support & 8 & 20 & 7 to 33 & 0.92 & 0.81 to 1 \\
\hline
\end{tabular}


TABLE 2: Reporting quality of details of needling from STRICTA $(n=40)$.

\begin{tabular}{|c|c|c|c|c|c|c|}
\hline Details of needling & & $\begin{array}{c}\text { No. of } \\
\text { positive trials }\end{array}$ & $\%$ & $95 \% \mathrm{CI}$ & $\begin{array}{l}\text { Cohen's } \\
\text { Kappa }\end{array}$ & $95 \% \mathrm{CI}$ \\
\hline $\begin{array}{l}\text { Acupuncture rational } \\
\text { Needling details }\end{array}$ & Reasoning for treatment provided & 38 & 95 & 87 to 100 & 0.66 & 0.50 to 0.81 \\
\hline Points used & Names or location of points used & 38 & 95 & 87 to 100 & 1 & - \\
\hline $\begin{array}{l}\text { Numbers of needles } \\
\text { inserted }\end{array}$ & $\begin{array}{c}\text { Number of needle insertions per } \\
\text { session }\end{array}$ & 17 & 43 & 27 to 59 & 0.85 & 0.73 to 0.97 \\
\hline Depth of insertion & Depth of insertion & 18 & 45 & 29 to 61 & 0.56 & 0.40 to 0.72 \\
\hline Responses elicited & $\begin{array}{l}\text { Responses sought (e.g., de qi or } \\
\text { muscle twitch response) }\end{array}$ & 19 & 48 & 32 to 64 & 0.37 & 0.21 to 0.53 \\
\hline Needle stimulation & $\begin{array}{c}\text { Needle stimulation (e.g., manual } \\
\text { or electrical) }\end{array}$ & 34 & 85 & 73 to 97 & 0.46 & 0.30 to 0.62 \\
\hline Needle retention time & Needle retention time per session & 38 & 95 & 88 to 100 & 1 & - \\
\hline Needle type & $\begin{array}{l}\text { Needle type (diameter, length and } \\
\text { manufacturer or material) }\end{array}$ & 27 & 68 & 53 to 83 & 0.38 & 0.22 to 0.54 \\
\hline \multicolumn{7}{|l|}{ Treatment regimen } \\
\hline Treatment frequency & Treatment frequency & 35 & 88 & 77 to 98 & 0.86 & 0.75 to 0.97 \\
\hline Course of treatment & Treatment course & 35 & 88 & 77 to 98 & 0.77 & 0.63 to 0.90 \\
\hline Practitioner background & Description of acupuncturists & 1 & 3 & 0 to 9 & 1 & - \\
\hline Control intervention & Precise description of the control or comparator. & 40 & 100 & - & 1 & - \\
\hline
\end{tabular}

Statistical Package for the Social Sciences (SPSS) version 20.0 .

\section{Results}

After searching, 34 Medline, 60 Embase, 1309 Cochrane Library, 13 WanFang, 129 VIP, and 1731 CNKI, totally 3276 recordings were retrieved (Figure 1). Among them, 1403 were English, and 1873 were Chinese. In accordance with the inclusion of the literature, excluding standards, and screening requirements, 40 RCT papers screened at last in Supplementary file 2 .

\subsection{Characteristics of Included Trials}

3.1.1. Year of Publication. In total, 40 RCTs were mainly published in the last 10 years including 1998 to 2019 (Figure 2). The interventions of them were manual acupuncture $(19,47.50 \%)$, electroacupuncture $(11,27.50 \%)$, scalp acupuncture $(1,2.50 \%)$ and fire needle $(9,22.50 \%)$, without ear acupuncture, sham acupuncture, or another intervention.

3.1.2. Journals and Languages. The 40 trials were published in 34 different journals. $11(27.50 \%)$ papers were published in general medical journals, $18(45.00 \%)$ papers in traditional or alternative medicine journals regarding journal category, $10(25.00 \%)$ in specialist medical journals, and 1 $(2.50 \%)$ paper was master's thesis. $2(5.00 \%)$ were published in Journal of New Chinese Medicine, 1 (2.50\%) in Acupuncture Research, and 1 (2.50\%) in the Shanghai Journal of Acupuncture Moxibustion. Only 1 paper (2.50\%) was published in the Complementary Alternative Medicine in English, and the remaining 39 (97.22\%) papers are all published in Chinese.
3.1.3. Funding Sources. Only 8 studies (20.00\%) reported their sources of funding. 3(7.50\%) received funding from national, $3(7.50 \%)$ from university or hospitals, and 2 (5.00\%) from regional, respectively. There is no report which received funding from pharmaceutical companies or research agencies in our sample.

3.2. Completeness of Reporting for the CONSORT Items. The data of reporting quality are listed in Table 1 based on the CONSORT statement. Good reporting were items "Background," "Randomised" in the title or "abstract," "Statistical methods," "Outcomes and estimation," and "Interpretation," with positive rates $>80 \%$. However, the reporting quality in items "Sample size," "Allocation concealment," "Implementation," "Blinding," "Flow chart," "Intent-to-treat analysis," "Ancillary analyses," "limitation," and "Clinical Trials Register" was very poor with positive rates $<10 \%$.

3.3. Completeness of Reporting for the STRICTA Items. The reporting quality ratings for the needling details are listed in Table 2. Based on the STRICTA guidelines, good reporting existed for the items "Acupuncture rational," "Points used," "Needle stimulation," "Needle retention time," "Course of treatment," and "Treatment frequency," with positive ratings $>80 \%$. However, the reporting quality of items "Numbers of needles inserted," "Depth of insertion," and "Responses elicited" was lower, with positive rates $<50 \%$, and "Practitioner background" was poor, with positive rates $<10 \%$.

The agreement between two reviewers of all items was observed in this study, and the agreement of mostly items was judged as moderate, substantial or good, except items "Implementation," "Responses elicited," and "Needle type" was assessed fair. 


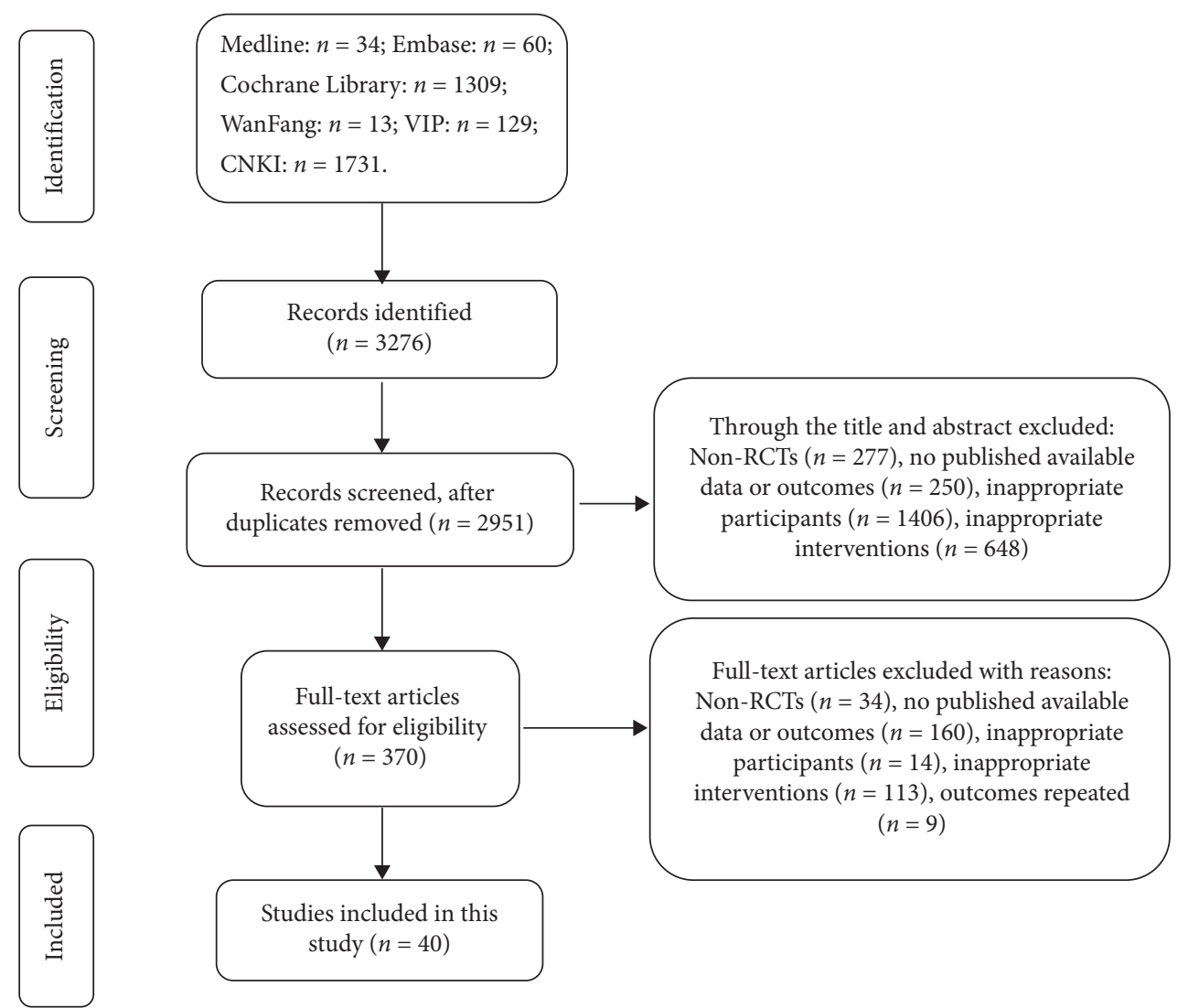

FIgURE 1: Flow chart of study selection of RCTs of acupuncture for acute HZ.

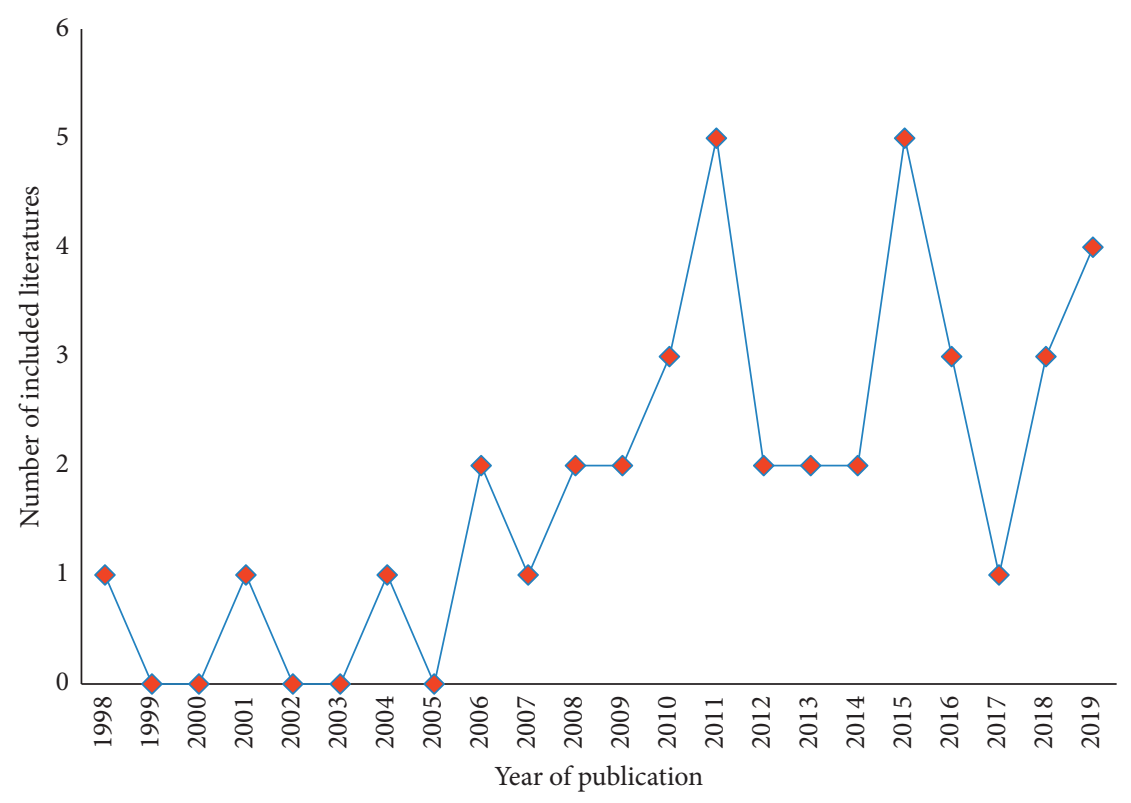

FIgURE 2: Number of RCTs of acupuncture for acute HZ published per year.

\section{Discussion}

This study has showed that the reporting quality of RCTs in acupuncture for $\mathrm{HZ}$ was dissatisfied. We need to pay attention as a lot of papers did not strictly comply with
CONSORT statement and STRICTA guidelines in this study. Low-quality clinical research influences other researchers to evaluate the study. Accurate and standardised reporting not only reduces the bias of systematic reviews but also contributes to medical decision-making. Acupuncturists can 
easily grasp effective operational procedures with highquality reporting.

In the 40 reports, most of them use "random," "random group," and other words simply to describe randomisation, and only 13 studies $(32.50 \%)$ report the method used to generate the random allocation sequence. Adequate randomisation is an effective measure to control the selective offsets and assure the authenticity of the results [21]. The researchers should use the scientific random sequence generation methods and describe it in detail to ensure the feasibility and repeatability of the study.

No literature referred allocation concealment, intent-totreat analysis, blinding of participants or care providers;only 1 paper $(2.50 \%)$ showed outcome assessors blinded after assignment to interventions. Research confirmed that allocation concealment, blinding, and intent-to-treat analysis are important protective measures to prevent implementation, measurement bias, and overestimate the effect especially in the evaluation of subjective outcome indicators such as pain, itching, and mood [22-24]. Therefore, these aspects need to be improved in later research.

The sample size estimation can avoid false negative results between intervention and control groups. There were only 2 reports $(5.00 \%)$ referred to the sample size estimates, and samples of 12 RCTs (30.00\%) were more than 100 . The lack of correct sample size estimation will lead to the lower test performance of RCT and affect the authenticity and reliability of the research results.

We select STRICTA guidelines to assess the quality of the "intervention" in report, as it has more detailed and specific reporting criteria for acupuncture. According to the STRICTA guidelines, the reporting quality of items "Numbers of needles inserted," "Depth of insertion," "Responses elicited," and "Practitioner background" was lower. A study indicates that the number of needles inserted and course of treatment were associated with outcomes in the treatment of pain [25]. Another study concluded that needling at correct acupoint locations was especially important to the effect of acupuncture for chronic pain [26]. However, there was little evidence to confirm the characteristics of acupuncture or acupuncturists have a wide variation of effects on pain at present, which required large sample sizes trials to evaluate. Therefore, it is significant to report all items of STRICTA guidelines.

Otherwise, there are several limitations in this study. Firstly, we did not search literature published in Japanese or Korean, and we only included trials published in English and Chinese. Secondly, we did not extract all items from the CONSORT 2010 statement and the STRICTA guidelines. Lastly, the literature finally included in this study is mainly in Chinese, and only one paper is in English.

\section{Conclusions}

The study indicates that the quality of reporting in RCTS on acupuncture for $\mathrm{HZ}$ was significantly lower and needs substantial improvement. In addition, most editors and researchers in Chinese are not familiar with the CONSORT statement and its extensions [16]. So, we recommend that the authors reporting and designing RCTs to abide by them and journal editors learn and raise the adoption of them to enhance the reporting quality of the RCTs in acupuncture.

\section{Data Availability}

The data used to support the findings of this study are included within the article and supplementary information.

\section{Conflicts of Interest}

The authors declare that they have no conflicts of interest.

\section{Authors' Contributions}

Guohua Lin and Pande Zhang conceptualized and designed the study. Yalin She and Zhenke Luo acquired the data. Wenya Pei and Kun Liu extracted the data. Liming $\mathrm{Lu}$ and Wenya Pei performed statistical analysis. Guifeng Qian and Jingchun Zeng carried out assessment of the reporting quality. Guifeng Qian and Jingchun Zeng wrote the manuscript. Guohua Lin and Pande Zhang revised the manuscript. All authors critically reviewed and commented on the manuscript. Guifeng Qian and Jingchun Zeng contributed equally to this work and are co-first authors.

\section{Acknowledgments}

This work was supported by the National Natural Science Foundation of China (no. 81574061), the Science and Technology Department of Guangdong Province (no. 2017A020213019), and the First Affiliated Hospital of Guangzhou University of Traditional Chinese Medicine (no. 2019QN23).

\section{Supplementary Materials}

The main features of the 40 included studies and their references in this paper. (Supplementary Materials)

\section{References}

[1] D. Cvjetkovic, J. Jovanovic, I. Hrnjakovic-Cvjetkovic, S. Brkic, and M. Bogdanovic, "Reactivation of herpes zoster infection by varicella-zoster virus," Medicinski pregled, vol. 52, no. 3-5, pp. 125-128, 1999.

[2] Y. Hama, K. Shiraki, Y. Yoshida et al., "Antibody to varicellazoster virus immediate-early protein 62 augments allodynia in zoster via brain-derived neurotrophic factor," Journal of Virology, vol. 84, no. 3, pp. 1616-1624, 2010.

[3] R. P. Insinga, R. F. Itzler, J. M. Pellissier, P. Saddier, and A. A. Nikas, "The incidence of herpes zoster in a United States administrative database," Journal of General Internal Medicine, vol. 20, no. 8, pp. 748-753, 2005.

[4] V. A. Morrison, G. R. Johnson, K. E. Schmader et al., "Longterm persistence of zoster vaccine efficacy," Clinical Infectious Diseases, vol. 60, no. 6, pp. 900-909, 2015. 
[5] T. Bharucha, D. Ming, and J. Breuer, "A critical appraisal of 'Shingrix', a novel herpes zoster subunit vaccine (HZ/Su or GSK1437173A) for varicella zoster virus," Human Vaccines \& Immunotherapeutics, vol. 13, no. 8, pp. 1789-1797, 2017.

[6] H. Wang, G. Yang, S. Wang, X. Zheng, W. Zhang, and Y. Li, "The most commonly treated acupuncture indications in the United States: a cross-sectional study," The American Journal of Chinese Medicine, vol. 46, no. 7, pp. 1387-1419, 2018.

[7] Z.-S. Liu, W.-N. Peng, B.-Y. Liu et al., "Clinical practice guideline of acupuncture for herpes zoster," Chinese Journal of Integrative Medicine, vol. 19, no. 1, pp. 58-67, 2013.

[8] T. Ursini, M. Tontodonati, L. Manzoli et al., "Acupuncture for the treatment of severe acute pain in herpes zoster: results of a nested, open-label, randomized trial in the VZV Pain Study," BMC Complementary and Alternative Medicine, vol. 11, no. 1, p. 46, 2011.

[9] M. E. Coyle, H. Liang, K. Wang et al., "Acupuncture plus moxibustion for herpes zoster: a systematic review and metaanalysis of randomized controlled trials," Dermatologic Therapy, vol. 30, no. 4, p. e12468, 2017.

[10] H. Tian and L. Wei, "[Filiform fire needling plus cotton moxibustion for 126 cases of herpes zoster]," Zhongguo Zhen Jiu, vol. 35, no. 10, pp. 1031-1032, 2015.

[11] C. Begg, M. Cho, S. Eastwood et al., "Improving the quality of reporting of randomized controlled trials. The CONSORT statement," JAMA: The Journal of the American Medical Association, vol. 276, no. 8, pp. 637-639, 1996.

[12] K. F. Schulz, D. G. Altman, and D. Moher, "CONSORT 2010 statement: updated guidelines for reporting parallel group randomised trials," BMJ, vol. 340, no. 1, p. c332, 2010.

[13] H. MacPherson, A. White, M. Cummings, K. Jobst, K. Rose, and R. Niemtzow, "Standards for reporting interventions in controlled trials of acupuncture: the STRICTA recommendations," Complementary Therapies in Medicine, vol. 9, no. 4, pp. 246-249, 2001.

[14] H. MacPherson, D. G. Altman, R. Hammerschlag et al., "Revised STandards for reporting interventions in clinical trials of acupuncture (STRICTA): extending the CONSORT statement," Journal of Evidence-Based Medicine, vol. 3, no. 3, pp. 140-155, 2010.

[15] L. Turner, L. Shamseer, D. G. Altman, K. F. Schulz, and D. Moher, "Does use of the CONSORT Statement impact the completeness of reporting of randomised controlled trials published in medical journals? a Cochrane review," Systematic Reviews, vol. 1, no. 1, p. 60, 2012.

[16] B. Ma, Z.-M. Chen, J.-K. Xu et al., "Do the CONSORT and STRICTA checklists improve the reporting quality of acupuncture and moxibustion randomized controlled trials published in Chinese journals? A systematic review and analysis of trends," PLoS One, vol. 11, no. 1, Article ID e0147244, 2016.

[17] K. H. Kim, J. W. Kang, M. S. Lee, and J.-D. Lee, “Assessment of the quality of reporting in randomised controlled trials of acupuncture in the Korean literature using the CONSORT statement and STRICTA guidelines," BMJ Open, vol. 4, no. 7, Article ID e005068, 2014.

[18] S. Svenkerud and H. MacPherson, "The impact of STRICTA and CONSORT on reporting of randomised control trials of acupuncture: a systematic methodological evaluation," Acupuncture in Medicine, vol. 36, no. 6, pp. 349-357, 2018.

[19] R. N. Werner, A. F. Nikkels, B. Marinović et al., "European consensus-based (S2k) guideline on the management of herpes zoster-guided by the European Dermatology Forum (EDF) in cooperation with the European Academy of
Dermatology and Venereology (EADV), Part 1: Diagnosis," Journal of the European Academy of Dermatology and Venereology, vol. 31, no. 1, pp. 9-19, 2017.

[20] R. Dworkin and R. Portenoy, "Proposed classification of herpes zoster pain," The Lancet, vol. 343, no. 8913, p. 1648, 1994.

[21] V. W. Berger and J. D. Bears, "When can a clinical trial be called 'randomized'?," Vaccine, vol. 21, no. 5-6, pp. 468-472, 2003.

[22] K. F. Schulz, I. Chalmers, R. J. Hayes, and D. G. Altman, "Empirical evidence of bias. Dimensions of methodological quality associated with estimates of treatment effects in controlled trials," JAMA, vol. 273, no. 5, pp. 408-412, 1995.

[23] L. Wood, M. Egger, L. L. Gluud et al., "Empirical evidence of bias in treatment effect estimates in controlled trials with different interventions and outcomes: meta-epidemiological study," BMJ, vol. 336, no. 7644, pp. 601-605, 2008.

[24] J. Savovic, H. Jones, D. Altman et al., "Influence of reported study design characteristics on intervention effect estimates from randomised controlled trials: combined analysis of meta-epidemiological studies," Health Technology Assessment, vol. 16, no. 35, pp. 1-82, 2012.

[25] H. MacPherson, A. C. Maschino, G. Lewith et al., "Characteristics of acupuncture treatment associated with outcome: an individual patient meta-analysis of 17,922 patients with chronic pain in randomised controlled trials," PLoS One, vol. 8, no. 10, Article ID e77438, 2013.

[26] A. J. Vickers, E. A. Vertosick, G. Lewith et al., "Acupuncture for chronic pain: update of an individual patient data metaanalysis," The Journal of Pain, vol. 19, no. 5, pp. 455-474, 2018. 